\title{
Aversive-Bias and Stage-Selectivity in Neurons of the Primate Amygdala during Acquisition, Extinction, and Overnight Retention
}

\author{
Uri Livneh and Rony Paz \\ Department of Neurobiology, Weizmann Institute of Science, Rehovot, Israel 76100
}

Extensive evidence implicates the amygdala as a major station for acquisition, extinction, and consolidation of emotional memories. Most of this work relies on fear-conditioning in rodents and imaging in humans. Few studies have explored coding of value in the primate amygdala, but the circuitry that underlies extinction and overnight retention remains largely unexplored. We developed a learning paradigm for nonhuman primates (macaca fascicularis) and recorded the activity of single neurons during the different stages of acquisition, extinction, and overnight consolidation of pleasant and aversive tone-odor associations. We find that many neurons become phase-locked to respiratory cycles in a stage-dependent manner, emphasizing the flexibility of amygdala neurons to represent the current state and change their spontaneous activity accordingly. We suggest that these changes can serve to increase neuronal sensitivity to an upcoming event and facilitate learning mechanisms. We further show formation of aversive-bias during the acquisition of associations and during overnight retention, in the sense that neurons preferentially code for the aversive conditioned stimuli, even if they initially homogenously represent value of the reinforcer. Our findings show flexible representations in the primate amygdala during the different cycles of learning and memory, and suggest selective potentiation of aversive information.

\section{Introduction}

The amygdala is a key structure mediating acquisition and regulation of emotional memories, and has been implicated in learning, retrieval, extinction and consolidation (LeDoux, 2000; Davis and Whalen, 2001; McGaugh, 2004). It receives parallel complex sensory inputs (McDonald, 1998), and its projections allow enhancement of emotional memories (McGaugh, 2004; Paz et al., 2006; Tsoory et al., 2008). Studies in rodents use fear-conditioning paradigms as a model (Fanselow and Poulos, 2005), with a wealth of physiological methods to delineate the underlying circuitry for acquisition and expression of memories in the amygdala (Maren and Quirk, 2004; Ciocchi et al., 2010; Haubensak et al., 2010; Pape and Paré, 2010). The modulation of fear by extinction is also mediated by circuits within the amygdala (Myers and Davis, 2007; Sotres-Bayon et al., 2007; Herry et al., 2008; Likhtik et al., 2008; Amano et al., 2010; Duvarci et al., 2011) which are activated from the ventral/medial-prefrontalcortices (v/mPFC; Milad and Quirk, 2002; Paré et al., 2004).

Accordingly, in humans, imaging studies have shown the importance of the amygdala in acquisition and maintenance

Received Jan. 23, 2012; revised March 15, 2012; accepted April 4, 2012.

Author contributions: U.L. and R.P. designed research; U.L. performed research; U.L. analyzed data; U.L. and R.P. wrote the paper.

This work was supported by Israel Science Foundation Grant 430/08, European Research Council reintegration and starting grants to R.P. We thank Yossi Shohat for invaluable contribution for the animals work and welfare, Dr. Eilat Kahana and Dr. Gil Hecht for help with medical and surgical procedures, and Dr. Edna Furman-Haran and Nachum Stern for MRI procedures.

Correspondence should be addressed to Dr. Rony Paz, Department of Neurobiology, Weizmann Institute of Science, Rehovot, Israel 76100. E-mail: rony.paz@weizmann.ac.il.

DOI:10.1523/JNEUROSCI.0323-12.2012

Copyright $\odot 2012$ the authors $\quad 0270-6474 / 12 / 328598-13 \$ 15.00 / 0$ of emotional memories and found an interplay with the vmPFC during extinction (Phelps et al., 2004), hence suggesting a role for these structures in anxiety disorders and posttraumatic stress disorder (PTSD; Delgado et al., 2006; Milad et al., 2006; Shin et al., 2006). Compared with rodents, primates exhibit a highly flexible control of emotions that is mediated by interactions between the amygdala and prefrontal cortices (PFC; Ochsner and Gross, 2005; Quirk and Beer, 2006), and this is achieved by rich and complex anatomical projections (Amaral and Price, 1984; McDonald, 1998; Ghashghaei et al., 2007). Few lesion studies have explored retention and extinction (Izquierdo and Murray, 2005; Antoniadis et al., 2009), and early electrophysiological work has addressed changes in affective significance of stimuli (Sanghera et al., 1979; Nishijo et al., 1988). However, recent studies have mainly focused on momentary representations of value: single neurons were shown to code contextual information regarding reward schedules (Sugase-Miyamoto and Richmond, 2005; Bermudez and Schultz, 2010), expectation and state value (Belova et al., 2007), dynamically switch responses with emotional valence (Paton et al., 2006), process facial expressions and identity (Gothard et al., 2007; Kuraoka and Nakamura, 2007), and track the skin-conductance-response (SCR) that reflects emotional state (Laine et al., 2009).

Hence, in contrast to the rich body of work that delineates the circuitry in the rodent amygdala, only few electrophysiological studies of the primate amygdala have addressed extinction and consolidation. Moreover, few have addressed directly the differences between positive and negative valence. To address this, we developed a paradigm of tone-odor conditioning (Livneh and 
Table 1. Summary of recorded neurons

\begin{tabular}{|c|c|c|c|c|}
\hline & Animal Z & Animal B & Animal L & Total \\
\hline \multicolumn{5}{|l|}{ Responsive neurons } \\
\hline Available neurons (at retention) $/ F R<0.3$ & $85(81) / 7$ & $145(131) / 14$ & $69(62) / 7$ & $288(274) / 28$ \\
\hline Responsive neurons (at retention) & $69(58)$ & $113(89)$ & $63(47)$ & 245 (194) \\
\hline \multirow[t]{2}{*}{ Breath-locked at Habituation/Acquisition/Extinction } & $39 / 50 / 41$ & $47 / 66 / 53$ & $25 / 39 / 29$ & $111 / 155 / 123$ \\
\hline & Aversive & Pleasant & Generalizing & Total \\
\hline \multicolumn{5}{|l|}{ Stage and valence selectivity } \\
\hline Acquisition neurons (FR Increase/Suppress) & $54(25 / 29)$ & $21(8 / 13)$ & $34(9 / 25)$ & $109(42 / 67)$ \\
\hline Extinction neurons & $34(17 / 17)$ & $34(14 / 20)$ & $32(12 / 20)$ & $100(43 / 57)$ \\
\hline Retention neurons & $23(14 / 9)$ & $21(9 / 12)$ & - & $44(23 / 21)$ \\
\hline
\end{tabular}

Paz, 2010). Odorants allow comparison between aversive and pleasant reinforcers within the same modality, gain direct anatomical access into the amygdala (Carmichael et al., 1994), and were shown to activate the human amygdala in learning tasks (Gottfried et al., 2002; Anderson et al., 2003). Here, we tested acquisition, extinction, and $24 \mathrm{~h}$ retention of aversive versus pleasant associations, and the dynamics of representation in single-neurons of the primate amygdala.

\section{Materials and Methods}

Animals. Three male macaca fascicularis $(4-7 \mathrm{~kg})$ were implanted with a recording chamber $(27 \times 27 \mathrm{~mm})$ above the right amygdala under deep anesthesia and aseptic conditions. All surgical and experimental procedures were approved and conducted in accordance with the regulations of the Weizmann Institute Animal Care and Use Committee (IACUC), following NIH regulations and with AAALAC accreditation. Food, water, and enrichments (e.g., fruits and play instruments) were available ad libitum during the whole period, except before medical procedures that require deep anesthesia.

MRI-based electrode positioning. Anatomical MRI scans were acquired before, during, and after the recording period. Anatomical images were acquired on a 3-tesla MRI scanner: (MAGNETOM Trio, Siemens) with a CP knee coil (Siemens). T1 weighted and 3D gradient-echo (MPRAGE) pulse sequence was acquired with TR of $2500 \mathrm{~ms}$, TI of $1100 \mathrm{~ms}$, TE of $3.36 \mathrm{~ms}, 8^{\circ}$ flip angle, and 2 averages. Images were acquired in the sagittal plane, $192 \times 192$ matrix and $0.8^{3} \mathrm{~mm}$ or $0.6^{3} \mathrm{~mm}$ resolution. A first scan was performed before surgery and used to align and refine anatomical maps for each individual animal (relative location of the amygdala and anatomical markers such as the interaural line and the anterior commissure). We used this scan to guide the positioning of the chamber on the skull at the surgery. After surgery we performed another scan with two electrodes directed toward the amygdala, and $2-3$ observers separately inspected the images and calculated the amygdala anterior-posterior and lateral-medial borders relative to each of the electrode penetrations. The depth of the amygdala was calculated from the dura surface based on the MRI at all penetration points. We used clear anatomical markers and visual similarity to identify the amygdala based on MRI images from primate atlas.

Recordings. The monkeys were seated in a dark room and each day, 3-4 microelectrodes (0.6-1.2 $\mathrm{M} \Omega$ glass/narylene-coated tungsten, Alpha Omega or We-sense) were lowered inside a metal guide (Gauge 25xxtw, OD:0.51 mm, ID:0.41 mm, Cadence) into the brain using a head-tower and electrode-positioning-system (Alpha Omega). The guide was lowered to penetrate and cross the dura and stopped $\sim 0.5-1 \mathrm{~cm}$ in the cortex. The electrodes were then moved independently further into the amygdala (we performed 4-7 mapping sessions in each animal by moving slowly and identifying electro-physiological markers of firing properties tracking the known anatomical pathway into the amygdala). Electrode signals were preamplified, $0.3 \mathrm{~Hz}-6 \mathrm{KHz}$ bandpass filtered and sampled at $25 \mathrm{Khz}$; and on-line spike sorting was performed using a template-based algorithm (Alpha Lab Pro, Alpha Omega). We allowed $30 \mathrm{~min}$ for the tissue and signal to stabilize before starting acquisition and behavioral protocol. At the end of the recording period, off-line spike sorting was further performed for all sessions to improve unit isolation (offline sorter, Plexon Inc).

Stimuli. Detailed descriptions of the odor delivery system (olfactometer) have been previously reported (Livneh and Paz, 2010). In brief, three hoses were attached to silicon made nasal mask held on the monkey's nose. The first hose deliver air into the mask at a constant flow. When stimuli is commanded, silent vacuum solenoids divert away the clean air and allow odorized air to be delivered into the mask. Importantly, the odorized air was delivered at the same pressure and flow as the clean air, and was commanded from outside the room to guarantee that the monkey would receive no cues regarding odor delivery. The second hose evacuate air from the mask at an equal flow to that delivered into the mask, and is responsible to the fast evacuation of the odors right after their release and maintenance of pressure within the mask. The third hose is connected to two pressure sensors with different sensitivity range ( $1 / 4$ " and 1 " $\mathrm{H}_{2} \mathrm{O}$ pressure range, AllSensors), that allowed measurement of respiratory behavior with minimal time lag. To load air with odor, filtered air flowed through Teflon made odor canister that contained either 1:20 solution of propionic-acid (Sigma-Aldrich) or 1:2 solution of banana-melon organic extract (Tevaoz). Both odors were diluted in mineral oil. Notice that propionic-acid has a high trigeminal component, and we previously tested our paradigm with hexanoic-acid as well with similar results (Livneh and Paz, 2010). Odors' concentration was tuned in preliminary experiments to obtain similar intensity by ratings of human subjects (as custom in olfaction studies, $p>0.1, t$ test).

Tones were $1000-2400 \mathrm{~Hz}$ pure sinus waves of $250 \mathrm{~ms}$ with $5 \mathrm{~ms}$ onset and offset ramps, generated with standard computer and delivered through a speaker (Adam 5 studio monitor, ADAM Audio) located 40 $\mathrm{cm}$ behind and to the center of the animal. All behavior was monitored and controlled by custom software written in LabView (National Instruments).

Behavior. Inhale onsets are detected in real-time using a double threshold algorithm implemented in LabView. Every $24 \pm 3$ breathing cycles a new trial was initiated by tone-emission. Pleasant and Aversive trials were pseudorandomly chosen but equalized in total number of presentations. Because odor release induces breath modulation that last up to 6 cycles (Livneh and Paz, 2010), we omitted all data acquired up to 10 cycles after any experimental stimuli for the evaluation of baseline volumes and spontaneous neuronal activity. Condition response (CR) was analyzed on modulations occurring $0-300 \mathrm{~ms}$ postinhale and unconditioned response (UR) was analyzed on modulations occurring $500-1000 \mathrm{~ms}$ postinhale onset.

Data analysis. Neurons with average firing rate (FR) of $<0.3 \mathrm{~Hz}$ were discarded from further analysis (28/327; Table 1). Phase locking of neurons to respiratory cycles was performed by constructing peristimulustime-histograms (PSTH) around onsets of baseline inhales. Warping was performed by normalizing spike times to the relative phase within the specific respiratory cycle in 24-bins. Rayleigh's test for circular uniformity $(p<0.05)$ determined for each neuron at each learning stages whether its activity is homogeneously distributed or not.

PSTH were computed for $2000 \mathrm{~ms}$ around events at each learning stage by using a $300 \mathrm{~ms}$ Gaussian window advanced at $25 \mathrm{~ms}$, and normalized by subtracting the mean and dividing by the SD of baseline activity from 
$1000 \mathrm{~ms}$ before the event. To compute overall spontaneous population activity (see Fig. 4), we inverted FR traces of cells that suppressed firing rate. Cells were defined as suppressing if the maximum deviation from preinhalation FR was negative. Finally, stimuli-evoked PSTH were normalized to baseline-inhale-response by subtracting the mean of the inhale-evoked response (taken from baseline inhales that were not preceded by an external event in 10 cycles) from the stimuli-evoked response. Notice this does not affect neurons without inhale related activity because the mean baseline trace of these neurons is zero.

Nonparametric Wilcoxon signed rank test was used to identify differences in activity taken from 100 to $800 \mathrm{~ms}$ poststimulus. Valenceselectivity was determined by taking the maximum absolute value of the mean corrected firing rate following aversive and pleasant CS. This approach selects differences from spontaneous (baseline) activity whether the neurons suppress or increase their response. Previous findings have shown that the primate and the rodent amygdala exhibit robust excitatory as well as inhibitory responses (Belova et al., 2007; Herry et al., 2008; Mosher et al., 2010) hence our approach identifies both types. The sign of the maximum deviation was used to determine whether a neuron suppressed or increased firing rate. We repeated all analyses using windows spanning from $100-500$ to $100-1000 \mathrm{~ms}$ poststimulus and selected the 100-800 window based on the accumulating statistical strength of the effects reported. However, the main effects reported here were all replicated using the different windows sizes. Functional neurons were computed from the total number of responsive neurons i.e., that change their FR to any of the stimuli (CS/US) at any of the behavioral stages (Wilcoxon signed rank test, $p<0.05$ ).

Trial-by-trial analysis. For each individual inhale during the acquisition stage we sought the 10 most similar baseline inhales, by finding those with the minimal Euclidean distance at $0-300 \mathrm{~ms}$ postinhale onset. We subtracted the mean response of the neurons from these 10 baseline inhales from the corresponding acquisition inhale. This provided us with a measure of the strength of the neural response that is not due to the breathing per se (e.g., the motor response). We then computed pairwise Spearman rank correlations between the strength of the neural response and the strength of the behavioral response measured as the volume of the tone-inhale. The neural responses of each individual neuron were binned into five groups according to the size of the corresponding behavioral response (i.e., 6 trials at bin $\times 5$ bins $=30$ trials). The neural response was $z$-scored and pooled together for population analysis.

\section{Results}

We designed a learning paradigm of tone-odor conditioning (Fig. $1 A, B$ ). Real-time detection of spontaneous inhalation onsets was used to trigger tones (tone-inhale) or odors (odorinhale). Two new tones were introduced every day [aversive/ pleasant conditioned stimulus (ACS/PCS)] and predicted delivery of either aversive or pleasant odor at the next inhale [aversive/pleasant unconditioned stimulus (AUS/PUS)]. Experimental sessions consisted of habituation to tones; acquisition of tone-odor associations; and extinction of these associations. Each session started with a retention test for the previous day tones. Overall, we recorded 316 single neurons from the right Basolateral amygdala (BLA) of three monkeys for which recording locations were confirmed according to MRI scans with a calibrating electrode performed before, during, and after recordings (Fig. 2, Table 1).

\section{Acquisition and extinction of pleasant and aversive tone-odor associations}

To validate implicit valence, we analyzed volume modulations of the odor-inhale (Fig. 3A). We observed a marked inhibition for the aversive odor (defensive behavior) and an augmentation for the pleasant odor (approach behavior, Fig. 3B; ANOVA, $p<$ 0.01 for both), and this was the case in $83 \%$ of the sessions $(63 / 76$, Fig. $2 B$, inset, $\left.p<0.01, \chi^{2}\right)$.
A Trial flow
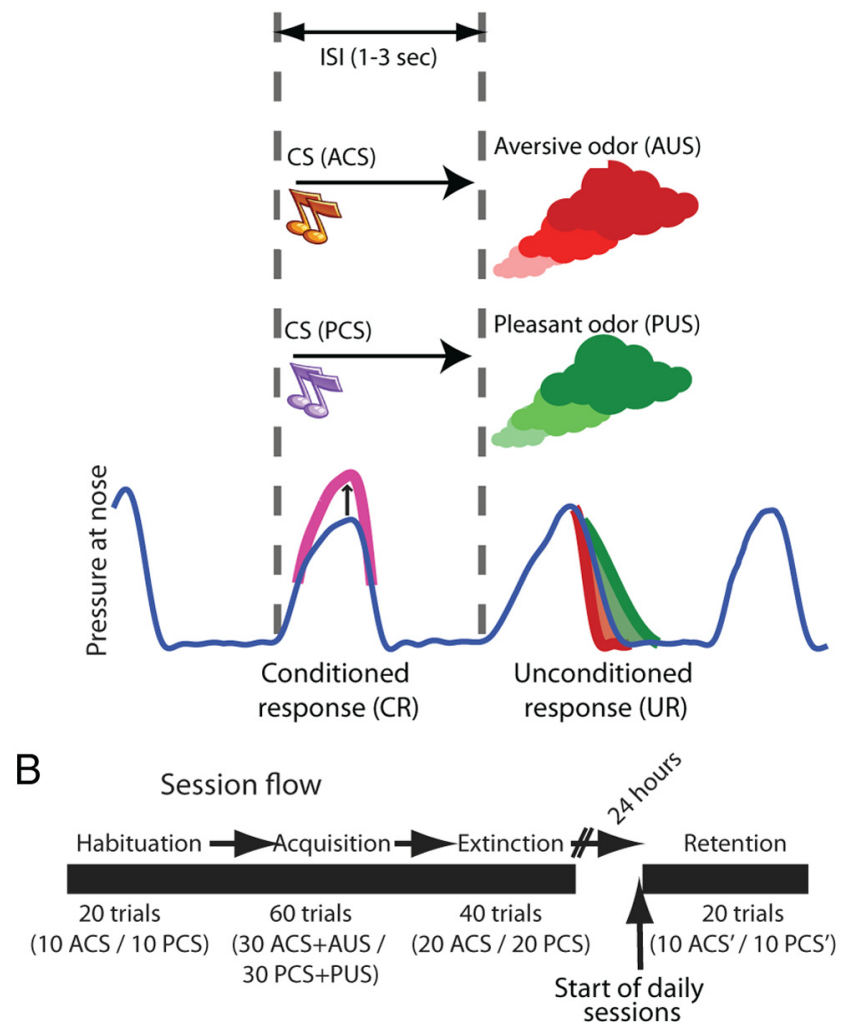

Figure 1. Behavioral paradigm. $\boldsymbol{A}$, Spontaneous breathing was continuously monitored (blue line) and inhalation onsets were detected online (dashed vertical lines). Tone initiation was triggered by onset of spontaneous inhalation and predicted odor release at the onset of the next spontaneous inhalation. Each daily session, an ACS was paired with AUS, and a PCS was paired with a pleasant odor PUS. Also shown is a typical UR which was an elevated inhalevolume for pleasant odor (green) and reduced volume for aversive odor (red); and a typical conditioned preparatory response (magenta, $C R$; a mimetic-response for the pleasant and compensatory-response for the aversive odor). $\boldsymbol{B}$, Each session started with a habituation stage where the two new tones were presented without odors (10 trials of each, randomly interleaved); followed by the acquisition stage described above $(30+30$ trials); and an extinction stage, where the two tones were presented again without reinforcers $(20+20$ trials). At the beginning of each session, we first tested for overnight retention of associations by presenting the tones from the previous day $(10+10$ trials).

Next, to examine learning of associations, we analyzed tone-inhale modulations and found them to be significantly augmented during acquisition (Fig. $3 C$ ), reflecting a conditioned preparatory response. Paired tones resulted in differential inhales across the different stages-elevated during acquisition when compared with habituation, and then returned to baseline during extinction learning (Fig. $3 D ; p<$ $0.01,2$-way ANOVA) - and this was also the case when considering each valence/tone separately $(p<0.05)$. Importantly, they were different between aversive and pleasant associations, an effect that occurred gradually during the acquisition stage (Fig. $3 D, E ; p<0.01,2$-way ANOVA). Further analysis indicated that valence differences developed by trials $12-30$ of the acquisition (Fig. $3 E ; p<0.01$, post hoc tests), and reflect the difference between mimetic responses to positive valence and compensatory responses to negative valence.

This demonstrates that the monkeys learned to associate tones with odors and their respective valence, and then learned to extinguish these associations. 


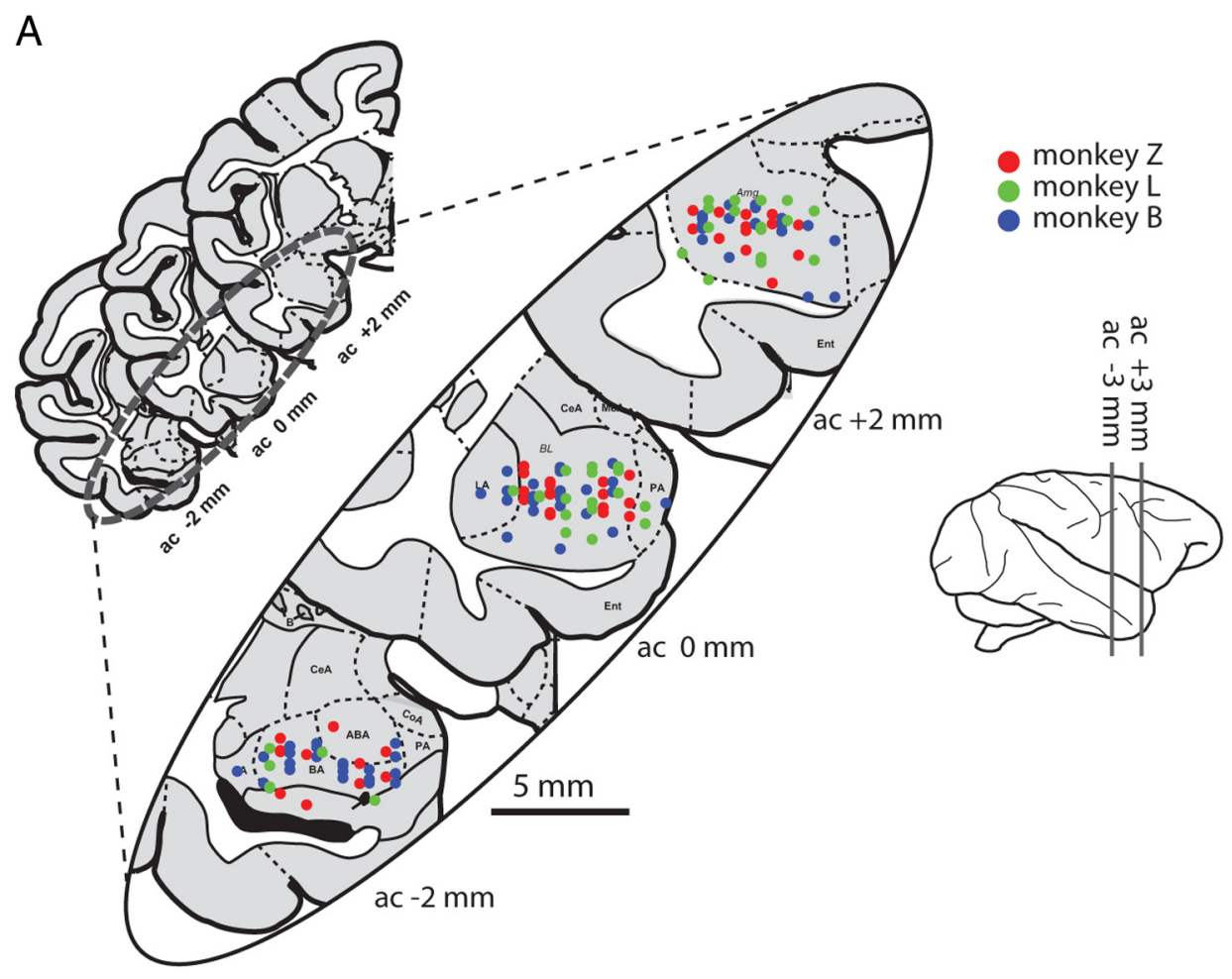

B

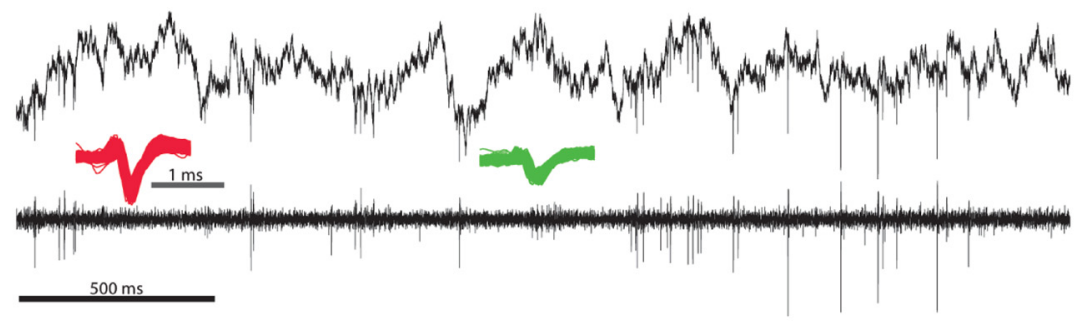

C
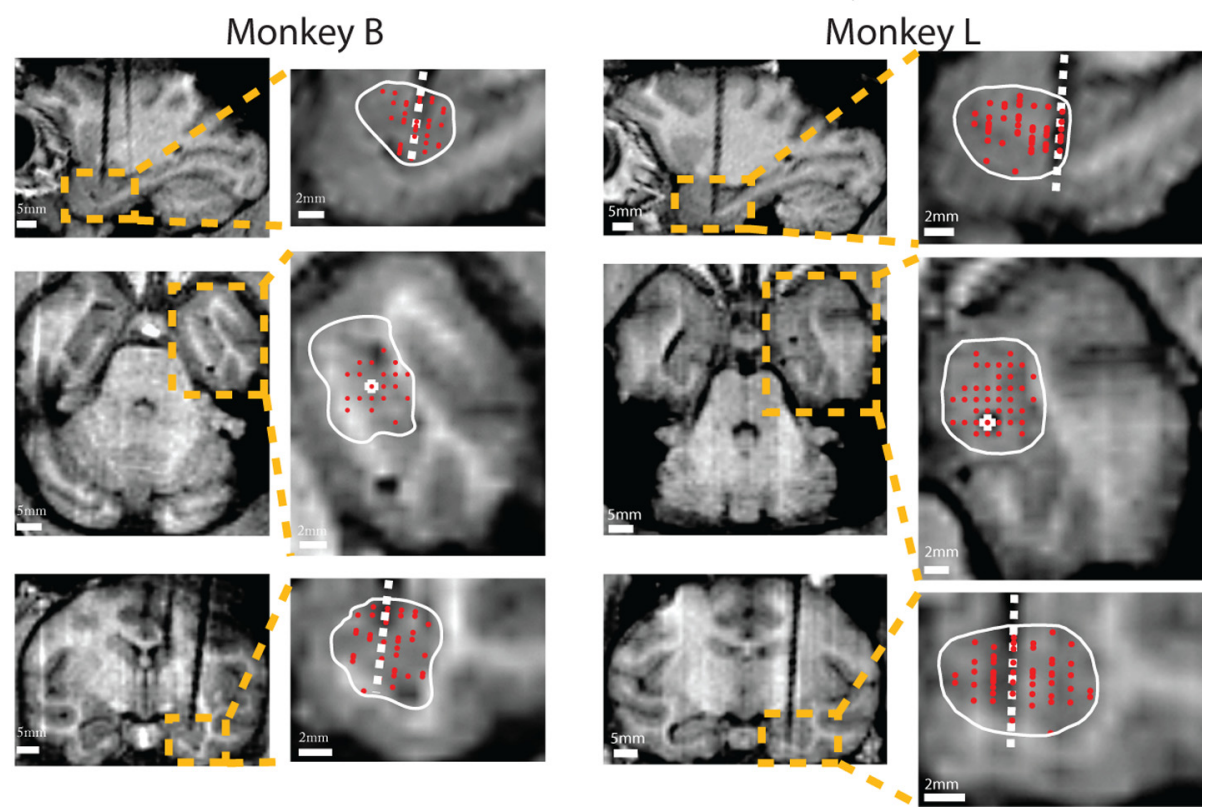

Figure 2. Reconstructed recording locations. A, Recording sites in the three monkeys are projected on the nearest of three coronal slices of the amygdala (from Martin and Bowden, 2000). $\boldsymbol{B}$, Sample raw extracellular signal (upper trace) and the bandpass filtered signal (bottom trace), with two representative spike waveforms (green and red). C, MRl images (first and third column), their magnification (second and forth columns) and the traces of calibrating electrode (white dashed line) in two monkeys. Amygdala borders (white line) are plotted on three perpendicular MRI planes with recording sites overlaid (red dots). MRI was performed before, during, and after the recording period with 2-3 localization electrodes for alignment. 
A

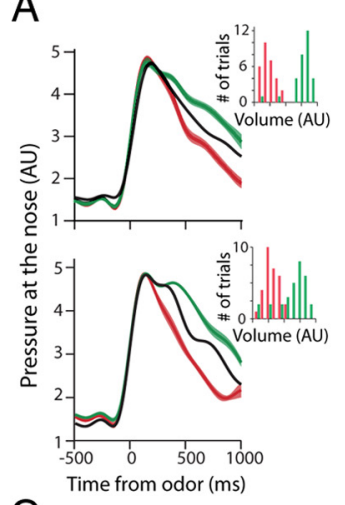

C

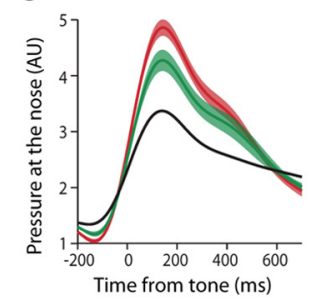

D

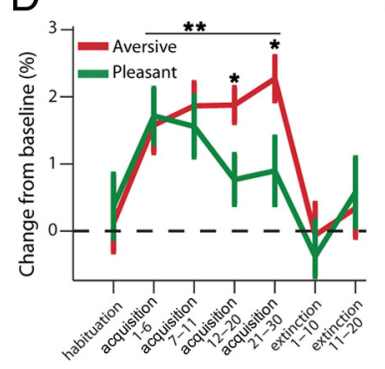

B
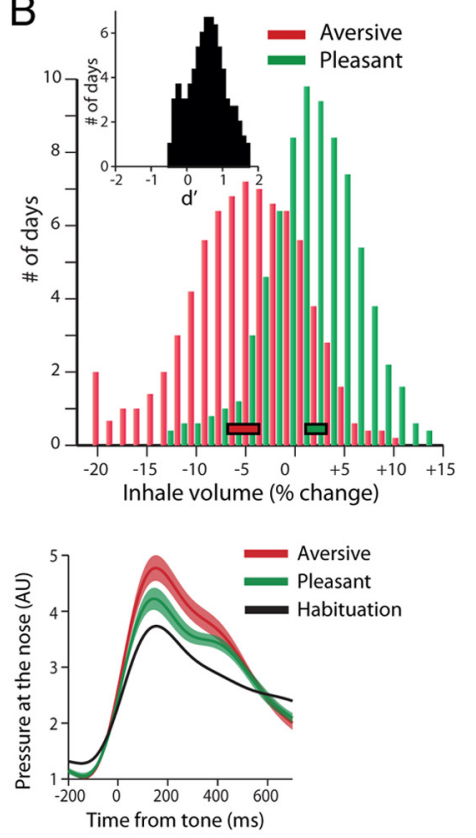

E

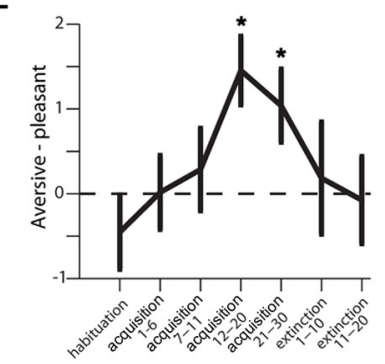

Figure 3. Valence is evident in UR; acquisition and extinction of valence is evident in CR. $A$, UR: release of aversive odor induced a defensive behavior: inhibition of inhale-volume (red) compared with baseline spontaneous inhales (black); and conversely, release of pleasant odor induced an approach behavior: augmentation of inhale-volume (green). Shown are averages from two daily sessions (top and bottom graphs) with corresponding histograms of all single inhalations' volume (insets, $p<0.01$ for both, $t$ tests). $\boldsymbol{B}$, Mean modulation of inhale-volumes following aversive/pleasant odor release. Rectangles at the bottom indicate Mean $\pm \operatorname{SEM}\left(p<0.01\right.$, ttest). Inset shows $\mathrm{d}^{\prime}$ comparing aversive and pleasant within each session, showing the effect is pronounced in most experimental days $\left(87 \%, p<0.01, \chi^{2}\right)$. Histograms smoothed with a 3-bin kernel for presentation only. C, CR: following acquisition, $(S$ tones induced augmented preparatory inhale-volume, and this was significantly higher for ACS (a compensatory-response) than for PCS (a mimetic-response, $p<0.05$, ttests). Shown are averages from two daily sessions. $\boldsymbol{D}$, Learning (CRs) developed during the acquisition and then extinguished ( $p<0.01$ for both learning-effect and valence-effect, two-way ANOVA). Extinction was not different from baseline ( $p>0.1, t$ test). $\boldsymbol{E}$, Differential valence effect evolved more gradually and was evident by trials $12-30$ of the acquisition ( $p<0.01$, posthoc $t$ tests), and returned to baseline during extinction.

\section{Stage-dependent neuronal responses are evident in spontaneous breath-locking}

To quantify neural responses that relate to a learning stage and that are not locked to specific stimuli, we used spontaneous inhalations that were not preceded by any stimuli (tone or odor) in the last 10 respiratory cycles as a baseline. This revealed neurons with periodic pattern of activity coupled to respiratory cycles (Fig. 4A). To align neural activity across breathing cycles, spike times were "warped" according to the duration of each individual cycle (Fig. 4B). Then, we tested separately whether spontaneous activity at habituation, acquisition or extinction is locked to respiratory cycles ( $p<0.05$, Rayleigh's test), and found that neurons are either locked or not, depending on the specific stage (Fig.
4C). Overall, a significant number of neurons exhibited spontaneous locking activity during all stages (Fig. $4 D$, habituation: $35 \%$ acquisition: $49 \%$; extinction: $38 \% ; 111 / 159 / 123$ cells respectively, $p<0.01$ for all, $\left.\chi^{2}\right)$, but many more did so during the acquisition ( $p<0.01$, ANOVA). Moreover, during the acquisition and extinction stages, but not in the habituation stage, most of the neurons had their peak firing rate at the first half of the respiratory cycle (i.e., during the inhalation phase; $p<0.01$, ANOVA).

In addition to the temporal locking, spontaneous inhalation onsets evoked stronger responses at the acquisition stage compared with habituation and extinction stages (Fig. $4 E, F ; p<0.01$, ANOVA, $p<0.01$, post hoc $t$ tests contrasting acquisition). We then compared tone-evoked to spontaneous-breathing-evoked responses (notice that in our paradigm, all stimuli are locked to breathing) and identified subpopulations of neurons that had a reduced or increased response to the stimuli when compared with breathing responses. Other subpopulations had toneevoked responses that can be fully accounted for by the spontaneous breathing activity (Fig. 4G). Hence, stimulus-evoked responses can be correctly identified only when compared with breathing-evoked responses, but not when compared with standard baseline (i.e., averaging prestimulus responses without taking breathing into account). We therefore normalize each stimuliresponse by the mean spontaneous inhalation response hereafter.

Together, these findings show that breathing-locked responses in the amygdala are abundant, but can be stage-dependent in individual neurons. This could be a consequence of the specific paradigm we use, emphasizing the flexibility of amygdala neurons to represent the current state and change their spontaneous activity accordingly (becoming locked to respiratory cycle in this case). These changes can serve to increase neuronal sensitivity to an upcoming event, and in parallel, facilitate cellular learning mechanisms.

\section{Different subpopulations selectively signal acquisition and extinction}

We next explored how neurons acquire discriminative responses for aversive and pleasant conditioned tones. To do so, we tested for neurons that discriminate between ACS-PCS responses during acquisition or extinction, but not during the habituation $(p<$ 0.05 , Wilcoxon-rank-sum test). Neurons were further classified according to the magnitude of their response compared with baseline as having aversive or pleasant selectivity (Fig. $5 A, B$ ). A third group of neurons exhibited CS response that was different in the acquisition or extinction when compared with the habituation, but did not discriminate between valence (PCS-ACS), and we refer to them as "generalizing" cells (Fig. $5 A$, B, bottom row). A total of 109 neurons were responsive during acquisition (44\% of responsive cells; acquisition-cells, $p<0.01$, binomial test) and 100 neurons were involved in extinction ( $40 \%$ of responsive cells; extinction-cells, $p<0.01$, binomial test). We found that the proportions of responding cells in the three different types of valence-selectivity during both acquisition and extinction were all above chance-level (Fig. $5 C, D ; p<0.05$, binomial test). However, there were significantly more aversive-selective cells during the acquisition stage (Fig. $5 C$; $p<0.01$, ANOVA), but a homogenous distribution for valence-selectivity during the extinction stage (Fig. $5 D ; p>0.1$, ANOVA).

We further identified two types of responses in all groups: increase in firing rates and decrease in firing rates in response to the tones (Fig. $6 A-D$ ), and compared response types during acquisition and extinction. Figure $6 E$ shows that there was little overlap between acquisition and extinction neurons for the three 
A
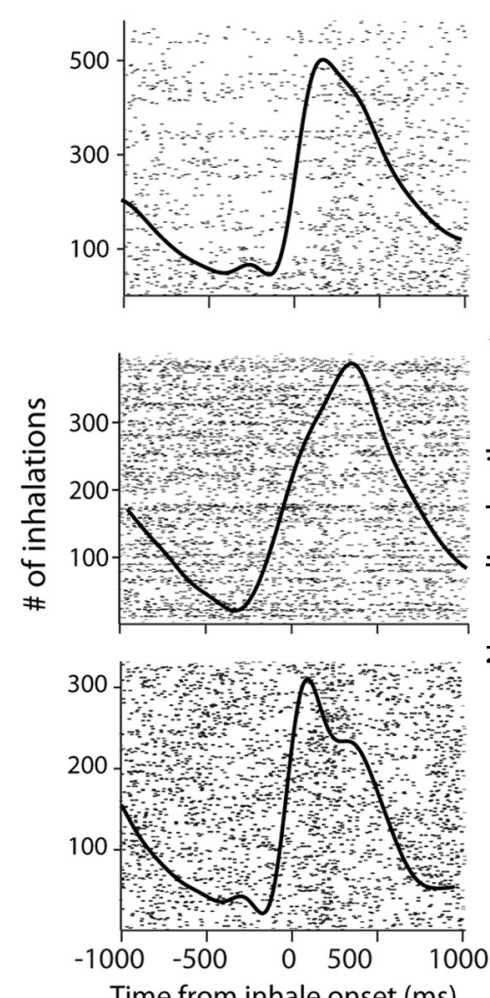

Time from inhale onset (ms)

D

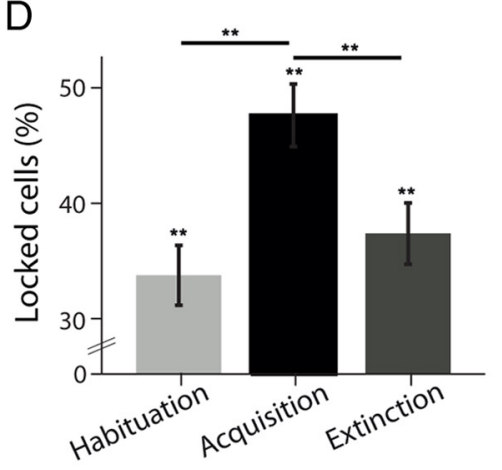

F

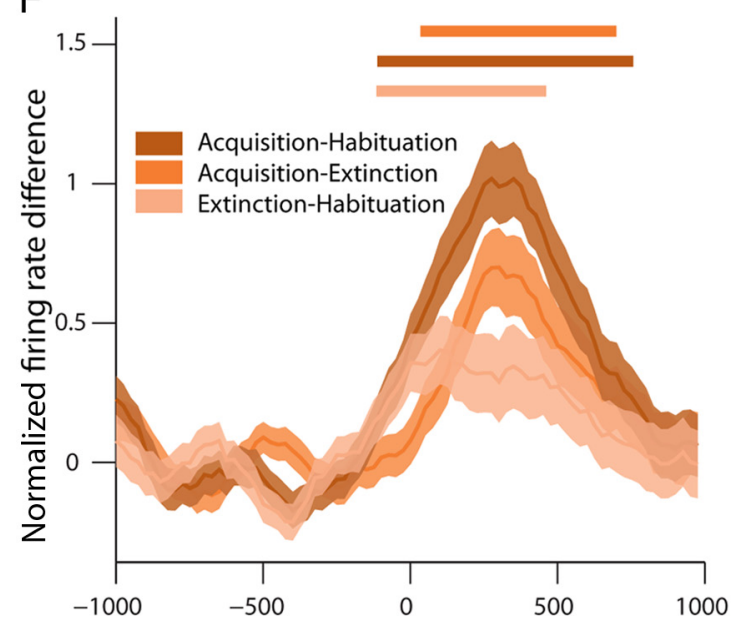

Time from inhale onset (ms)
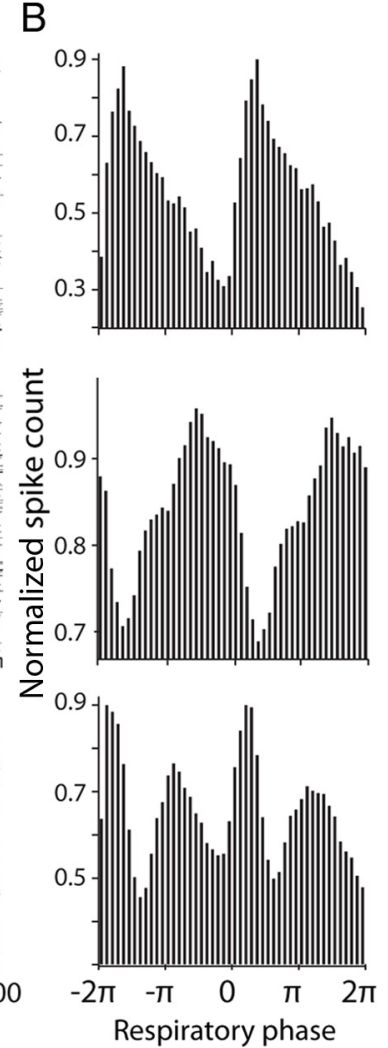

E

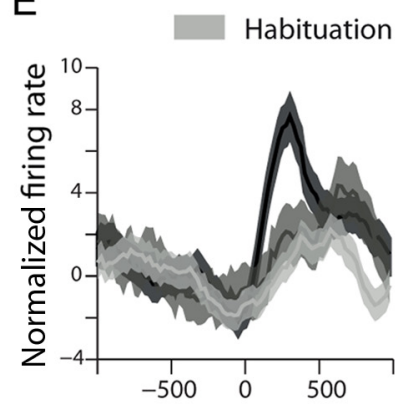

C
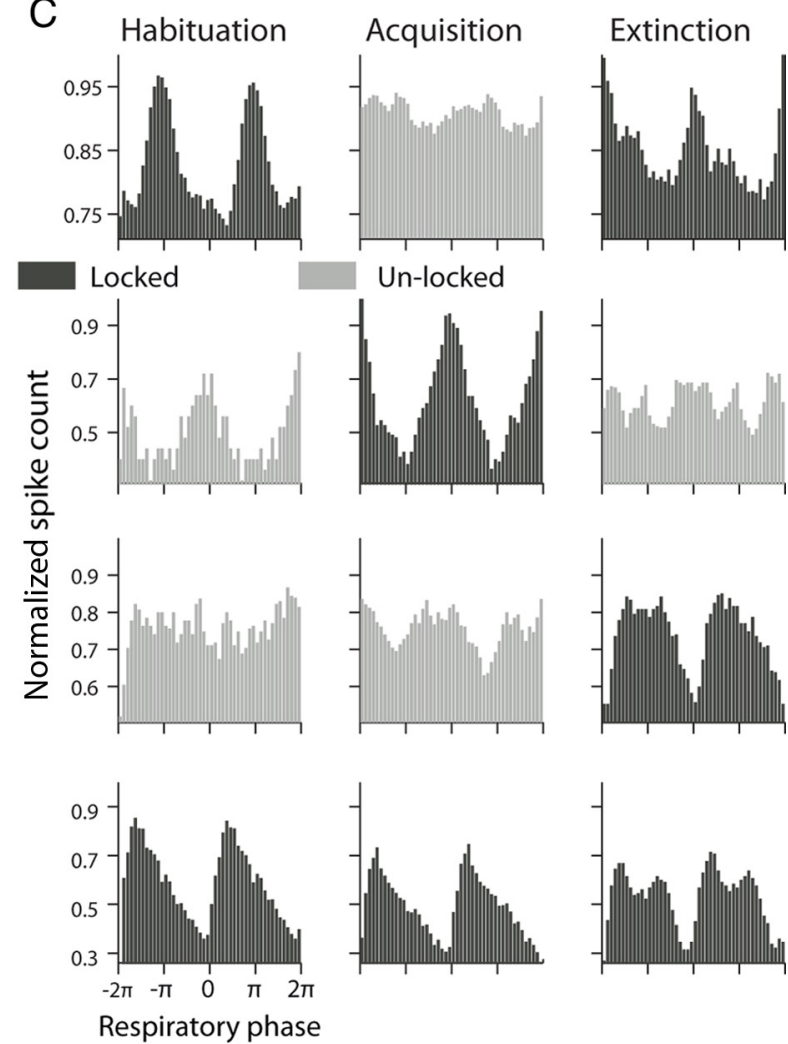

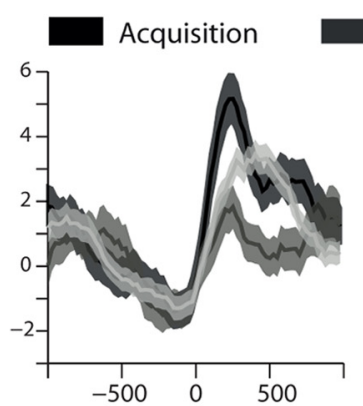

Time from inhale onset (ms)

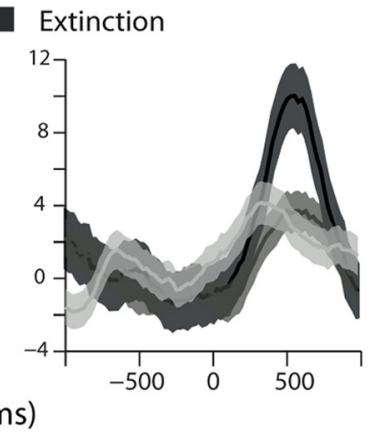

G

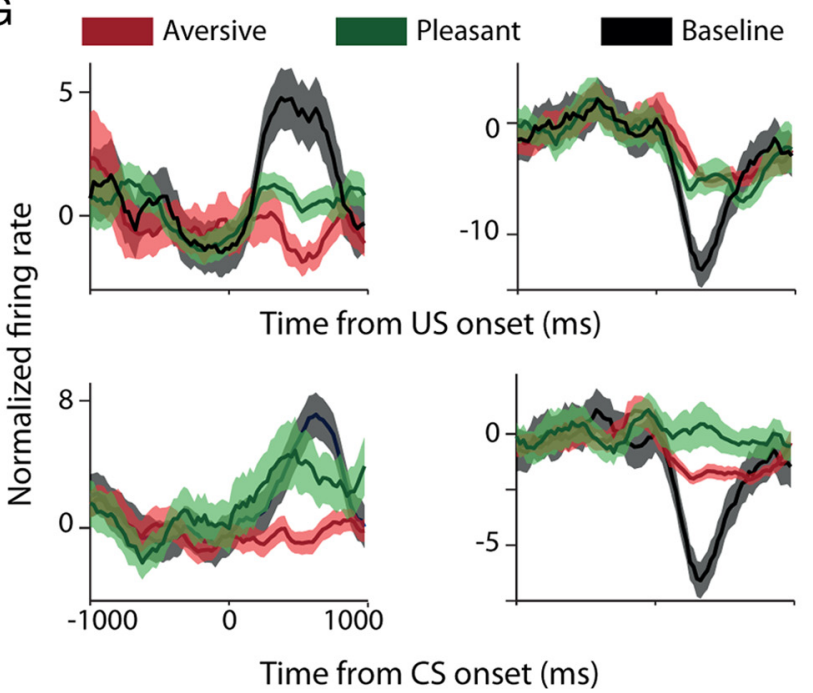

Figure 4. Amygdala neurons are locked and synchronized to respiration in a stage-dependent manner. $\boldsymbol{A}$, Raster plots of three single cells (rows) around onset of inhale (black line). $\boldsymbol{B}$, Phase-locking of these neurons revealed by computing PSTH after warping spike times in each individual respiratory cycle to $2 \pi$. C, Phase locking of four cells (rows) during (Figure legend continues.) 


\section{A}

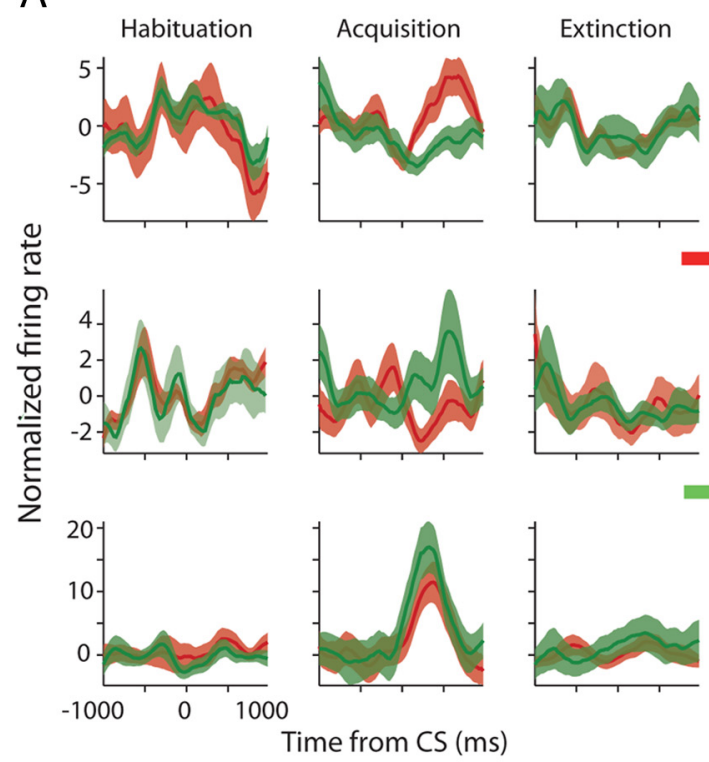

C

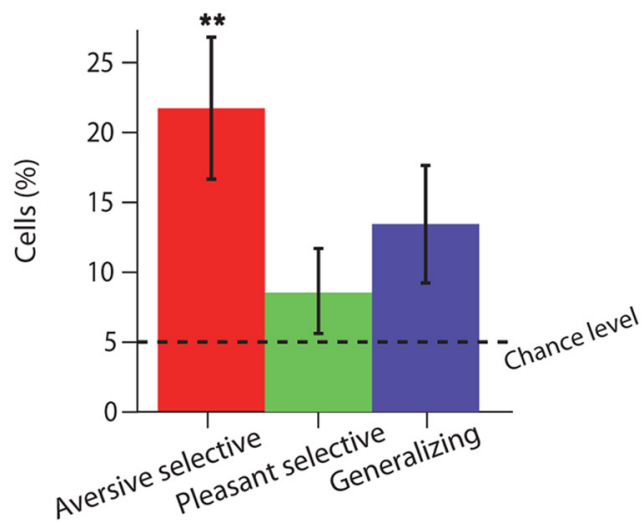

B

\section{extinction neurons}

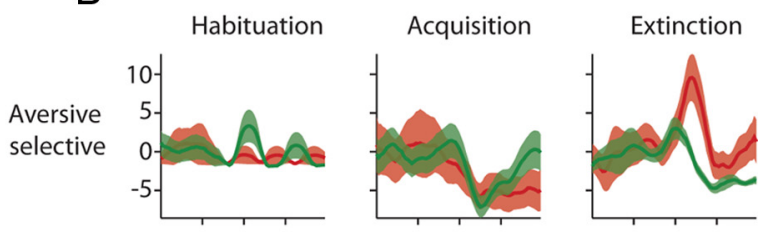

Aversive CS

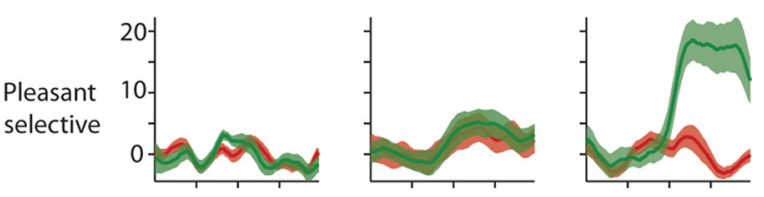

Pleasant CS
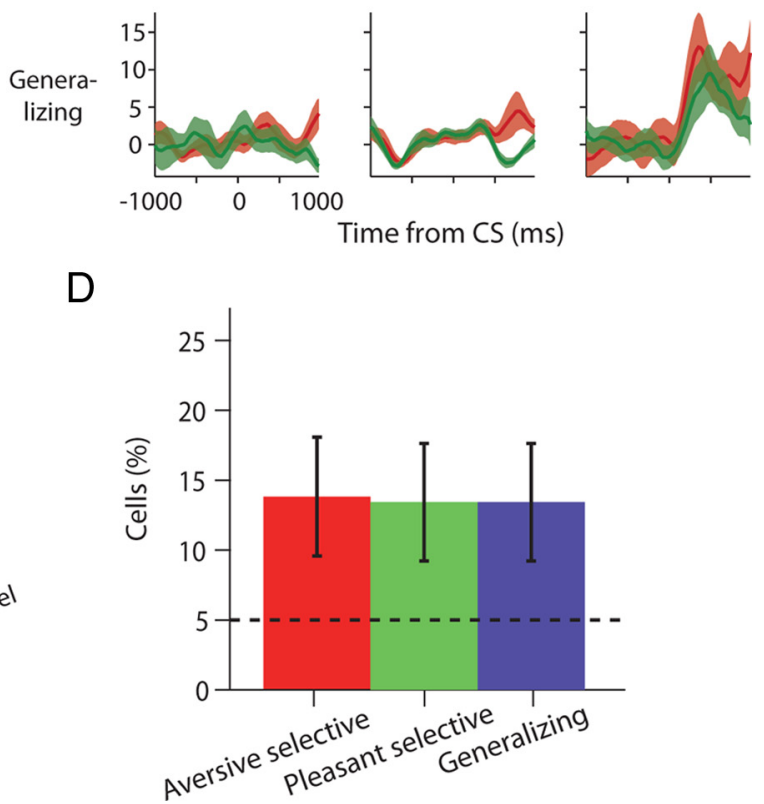

Figure 5. Neurons acquire differential responses to valence during both acquisition and extinction. $\boldsymbol{A}$, Acquisition neurons had selective tone responses (each row is one neuron, average response over trials \pm SEM in shaded color) in the acquisition when compared with habituation (columns), and had either aversive-selectivity (top row), pleasant- selectivity (middle row), or generalizing across valence (bottom row). B, Similarly for extinction neurons. See Results, Different subpopulations selectively signal acquisition and extinction, for definition and statistics used to identify and categorize neurons. $\boldsymbol{C}, \boldsymbol{D}$, Proportions of acquisition $(\boldsymbol{C})$ and extinction $(\boldsymbol{D})$ cells for the three types of responses ( $\pm 95 \%$ binomial confidence intervals). There were significantly more neurons than chance level in all groups ( $p<0.05$, binomial tests). There were significantly more aversive-selective neurons during acquisition, compared with all other groups ( $p<0.01$, AN0VA).

types of valence-selectivity (aversive, pleasant, and generalizing). In fact, the proportion of neurons that responded during both learning stages was not different from chance level $\left(\chi^{2}, p>0.1\right)$. This suggests that populations of aversive, pleasant and generalizing cells are largely independent for acquisition and extinction.

(Figure legend continued.) the different behavioral stages (columns). Black shows stages where the neuron was significantly locked ( $p<0.05$, Rayleigh test), and gray when it was not. $D$, Proportions of phase-locked neurons were significantly higher than chance level in all stages $\left(35 \%, 49 \%, 38 \%\right.$ correspondingly; $p<0.01$ for all, $\left.\chi^{2}\right)$, and the proportion during acquisition increased significantly and then returned to baseline during extinction $\left(p<0.01, \chi^{2}\right) . E$, In addition to the phase-locking, cells exhibited a higher firing rate during the inhalation phase of the cycle at the acquisition stage (notice that phase-locking potentially contributes, but not necessarily). $\boldsymbol{F}$, This was stronger in acquisition than in habituation or extinction. Shown is the difference in firing rate between the three possible combinations of behavioral stages, averaged over all neurons (lines represent significant bins, $p<0.05$, t tests). $G$, Stimulus presentation could result in suppression of evoked activity that can be revealed only when compared with baseline activity for spontaneous inhales. Shown are four representative neurons: left column, a decrease relative to spontaneous cycles; right column, an increase; upper row, US responses; lower row, $\mathrm{CS}$ responses.
Further validating this finding, plotting the response of acquisition neurons during extinction and vice versa indicates that neurons carry little information for the other learning stage (Fig. 6B, $C, E$ ).

Neural responses are correlated with trial-by-trial behavioral learned responses

We tested whether the acquired changes in neural activity to the CS correspond to the instantaneous magnitude of the trial-bytrial behavioral learned response (CR). Importantly, we made sure that the acquired neural responses do not reflect the inhalation, but reflect learning. To do so, we sorted tone-inhales during the acquisition stage (CRs) according to their size, and then located and matched for each tone inhale the 10 most similar baseline spontaneous inhalations (Fig. 7A). Neural responses during the acquisition were then corrected to the baseline responses, resulting in a neural measure that is not due to respiration per se. The corrected neural response of 64 cells significantly differed from zero for the aversive CS (24\% of responsive cells, $t$ test, $p<$ $0.05)$, indicating that these cells reliably modified their response to the CS, even when breath related activity is taken into consid- 
A

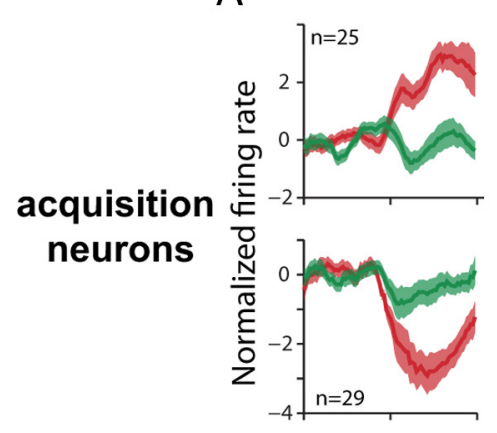

C

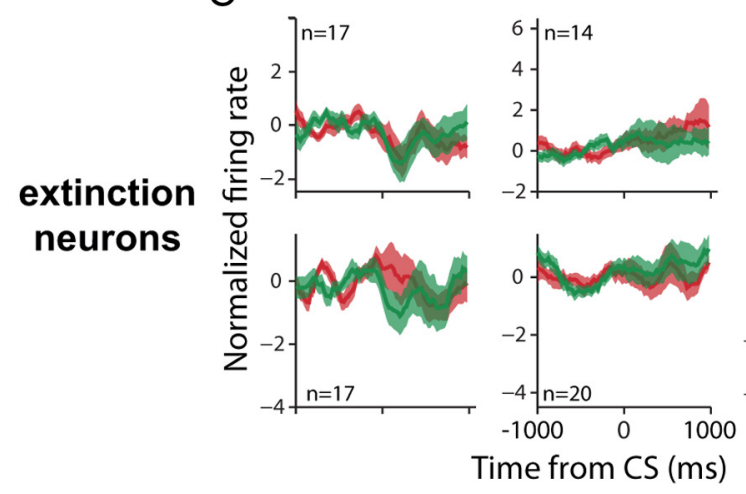

E

acquisition
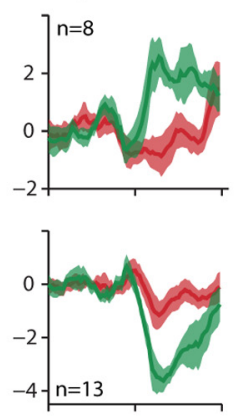

Aversive
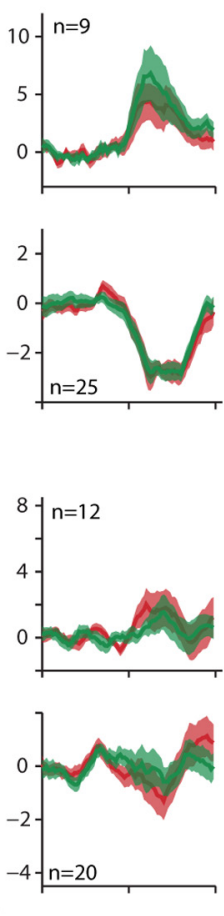

B

D
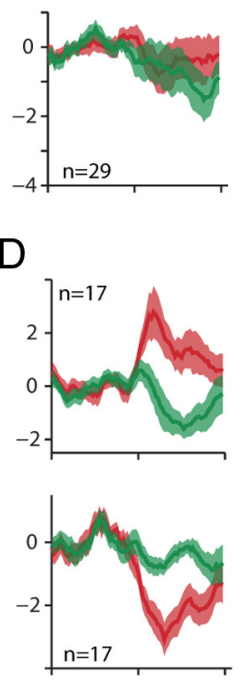

extinction
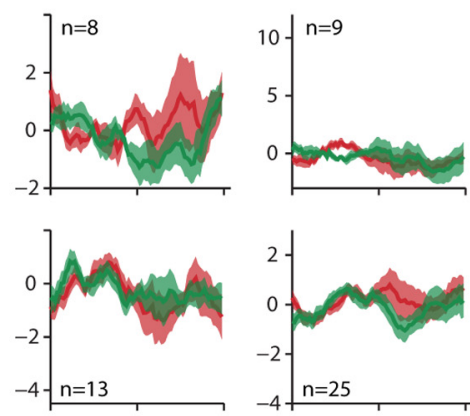

Pleasant
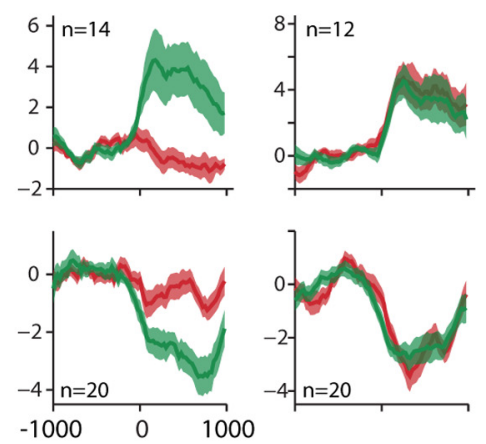

Time from CS (ms)

\section{Generalizing}

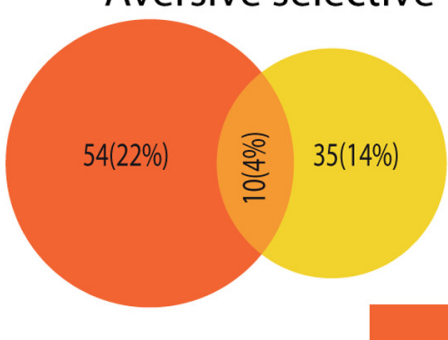

Pleasant selective
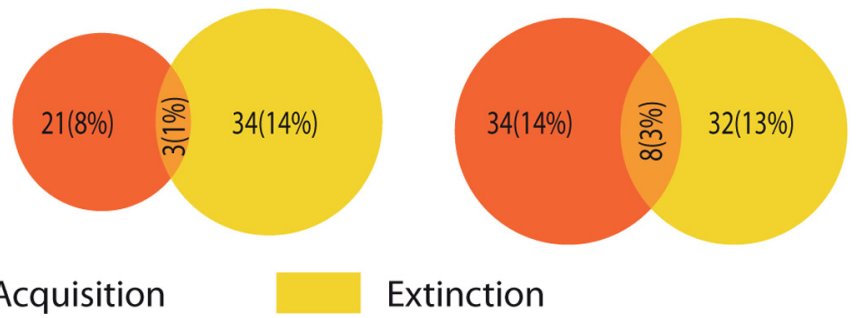

Extinction

Figure 6. Distinct populations are recruited during acquisition and extinction. $A-D$, Population means ( \pm SEM in shaded color) of all neurons after categorization based on whether they are aversive-selective, pleasant-selective (middle column) or generalizing (last column), and whether they respond during acquisition ( $\boldsymbol{A}$ ) and/or extinction (D) compared with habituation. To emphasize the richness of responses, we further separate neurons that increased their rate (top row) from neurons that decreased their rate (bottom row). Population means ( \pm SEM) for the acquisition neurons during extinction $(\boldsymbol{B})$ and for the extinction neurons during the acquisition $(\boldsymbol{C})$, suggest that the response of both group is limited to either the acquisition or the extinction. Notice that selection of neurons for $\boldsymbol{A}, \boldsymbol{B}$ was done without criteria for extinction, and for neurons in $\boldsymbol{C}, \boldsymbol{D}$ without criteria for acquisition; hence the lack of responses suggest that neurons carry very little information for the other stage. $E$, Few neurons had significant tone responses during both acquisition and extinction. The area of each circle and the intersection correspond to the number of neurons in each group (exact numbers are mentioned). All three intersections were not significantly different from expected $\left(p>0.1, \chi^{2}\right)$, indicating that distinct subgroups participate in acquisition and extinction.

eration. This shows that CS related activity is not driven by the conditioned response alone.

Next, we tested whether this corrected response holds more detailed information about the strength of the memory. The neural activity of $15 \%$ of the responsive cells significantly correlated with the strength of the preparatory response on a trial-by-trial basis $(p<$ $\left.0.05, \chi^{2}\right)$, with most of them having positive correlations (30/38; $79 \%$, Fig. $7 B$ ). This was also true at the population level, as corrected firing rate was significantly correlated with the magnitude of the preparatory response when pooling all neurons with positive and significant correlation (Fig. 7C, $r=0.8, p<0.001$ ) but also when pooling responses of all available neurons (Inset in Fig. 7C, $r=0.19$, $p<0.01$ ). Similarly, when we repeated the same analysis for the pleasant trials we identified that the corrected activity of $11 \%$ (27) of the cells was correlated with the strength of the behavioral response at these trials. There was little overlap between the pleasant and the aversive related neurons (11 neurons had significant correlation for both).

Similar proportions were found for the extinction stage with $34(14 \%)$ neurons having significant correlation with the behavioral response, and only 8 of these cells were correlated during acquisition as well. These results strengthen the findings that functionally distinctive subpopulations of neurons exist in the amygdala, and that these neurons represent the acquired response on top of the transient behavioral response.

\section{Independent representation of valence for CS and US reveals} an aversive bias

We identified aversive-selective, pleasant-selective and generalizing cells in a similar way as described above for the CS, but this 

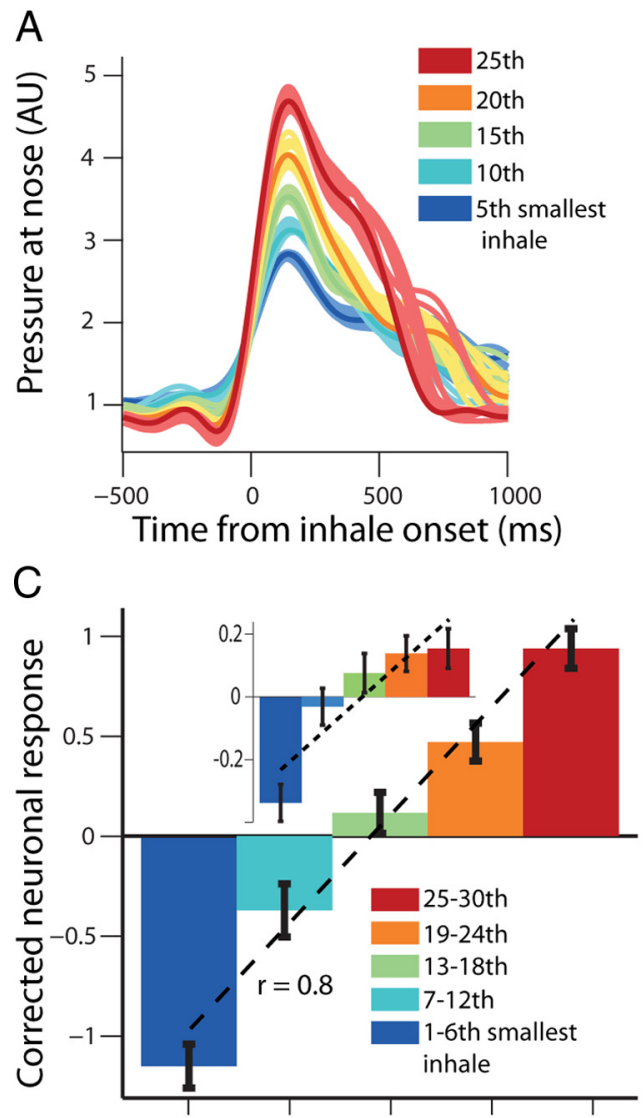

Strength of CS response
B
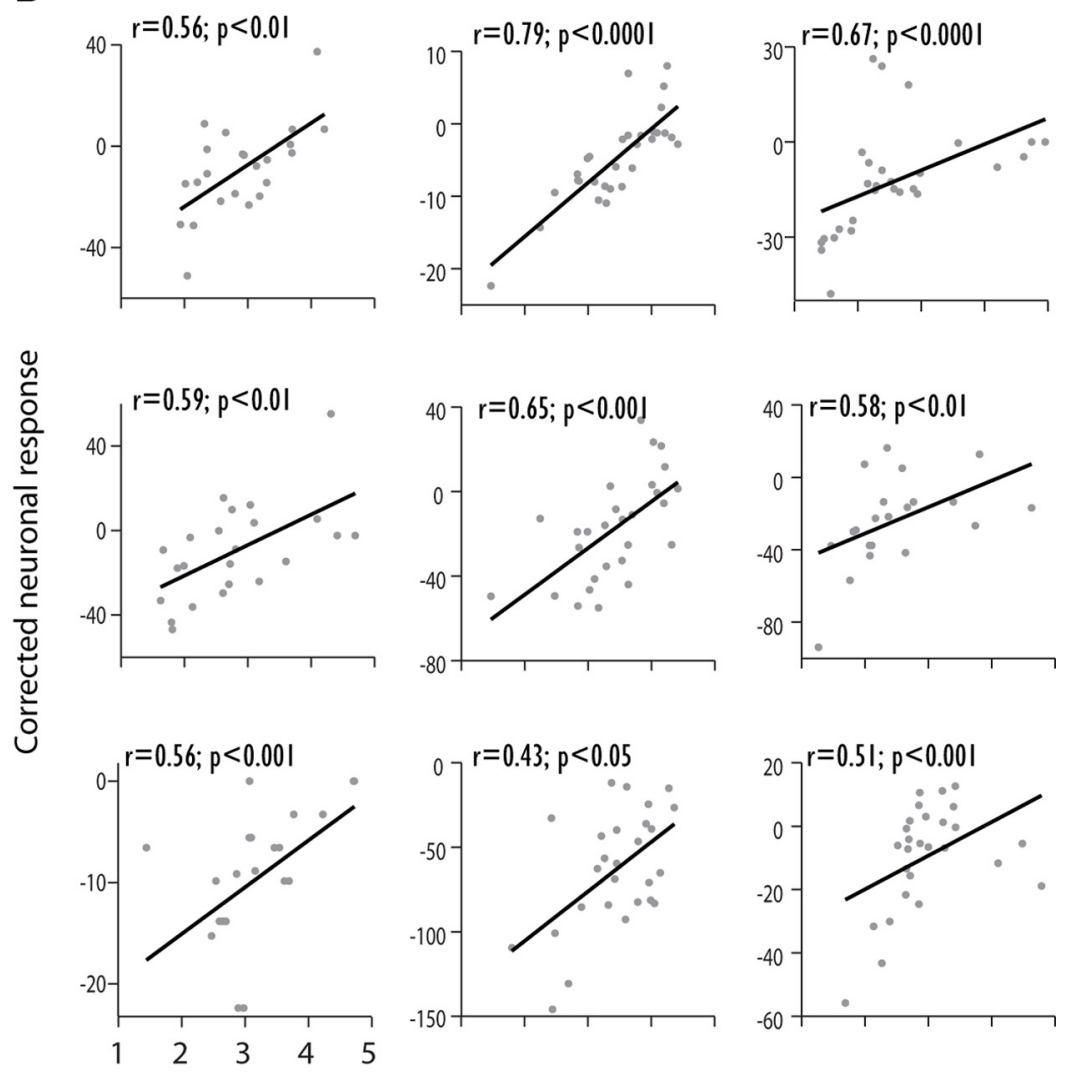

Strength of CS response

Figure 7. Preparatory neural responses indicate trial-by-trial learning. $\boldsymbol{A}$, We matched 10 spontaneous inhales with the minimal Euclidian distance to each single-trial CR (preparatory behavioral response). Shown are five different CRs (solid line of 5 different sizes for easier presentation) from one session overlaid with their matched spontaneous controls (thin line shaded color). Neural responses from these controls were used to correct the neural response for the CS on a trial-by-trial basis, hence removing the portion of the response that belongs to the behavior per se (e.g., motor-inhale). $\boldsymbol{B}$, trial-by-trial correlations of behavioral response (CR) with corrected (S-evoked neural response for 9 neurons (Spearman-correlation and $p$ values are shown on top). $\boldsymbol{C}$, CRs from all trials and sessions were pooled to five bins according to relative size, and correlated with neural responses averaged across all cells with positive correlation. This revealed a significant relationship ( $r=0.8, p<0.001$, Pearson) between neuronal response and learning that is independent of the pure behavioral response. The relationship is significant also when using all available cells (inset, $r=0.18, p<0.01)$.

time for the US (odor release, Fig. $8 A$ ). Overall, $57 \%$ of responsive neurons had odor-driven responses $(p<0.01$, binomial test). In these neurons, we found that $54 \%$ of the US aversiveselective neurons $\left(32 / 59, p<0.01, \chi^{2}\right), 42 \%$ of the US pleasantselective neurons $\left(20 / 48, p<0.01, \chi^{2}\right)$, and $38 \%$ generalizing US neurons $\left(14 / 37, p<0.01, \chi^{2}\right)$ became CS-responsive during the acquisition stage. These ratios were much higher than chancelevel, as indicated, but similar to the ratios of responding acquisition-cells, i.e., CS-responses $\left(p>0.1, \chi^{2}\right)$. However, whereas most of the acquisition responses of the US generalizing neurons were of the generalizing type, i.e., responding to both aversive and pleasant valence (Fig. $8 B$; $p<0.05$, ANOVA); most of the CS responses of the US valence-selective cells (i.e., pleasant or aversive) were of the aversive type (Fig. 8 B; ANOVA $p<0.01$ for both). To validate this at the population level, we computed a valence-selectivity index for the US response and compared it to the CS valence-selectivity index. This revealed a mild, yet significant, overall tendency for aversive CS coding (Fig. $8 C ; p<0.05$, $t$ test). To account for intensity, we identified sessions in which the absolute magnitude of breath modulation was similar for the aversive and the pleasant odors ( $t$ test, $p>0.05 ; 35 / 76$ sessions). A total of 125 cells were recorded in these days, and when inspected separately, the aversive bias reported above was still pres- ent ( $p<0.05, \chi^{2}$ for both), suggesting that intensity is not the only factor contributing to the valence-bias.

These results suggest that the amygdala slightly favors aversive coding during learning, even when it receives equivalent input regarding a pleasant reinforcer. It also shows the flexibility of coding and plasticity, as neurons can respond to a reinforcer yet acquire responses to a stimulus that predicts a reinforcer with the "opposite" valence.

\section{Overnight formation of aversive-bias}

At the beginning of each daily session we performed a retention test for the two tones that were conditioned and extinguished on the previous day. Hence, this was a 24 h overnight retention test. We compared the CR of aversive tone (volume of tone-inhale, as described above) to that of pleasant $\mathrm{CR}$ and found them to be significantly bigger (Fig. $9 A, B$; $p<0.01, t$ test). We verified that this is due to an increase response to the aversive tone rather than decrease to the pleasant, by normalizing the CRs by the tone-evoked inhale-volume of the habituation session that followed ( $p<0.01, t$ tests).

To compare valence relationships between retention and acquisition, we compared neural selectivity of valence during the retention test to the selectivity during the following acquisition 
A

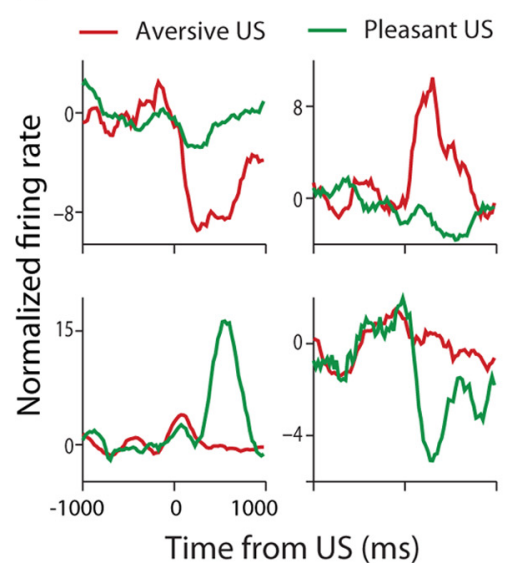

B

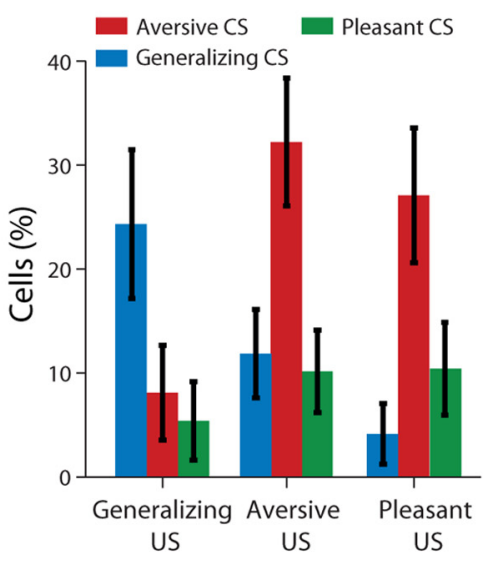

C

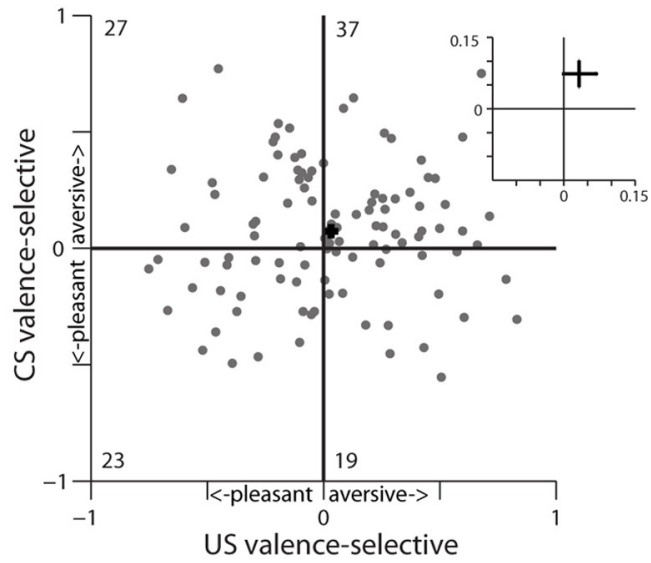

Figure 8. Contingency and aversive-bias in CS-US relationships. $\boldsymbol{A}$, Four representative neurons and their US (odor) responses (clockwise from upper-left corner: aversive-decreasing, aversiveincreasing, pleasant-decreasing, and pleasant-increasing). $\boldsymbol{B}$, Cells were classified as aversive, pleasant, or generalizing based on their US responses and then tested for their (S selectivity. Generalizing cells largely remained generalizing, whereas most aversive and pleasant US cells became aversive CS cells $\left(p<0.01, \chi^{2}\right)$. C, Plotting valence-selectivity index for US versus CS responses of all cells reveals a mild but significant aversive-bias (inset shows mean $+\mathrm{SEM}, p<0.05, t$ tests).

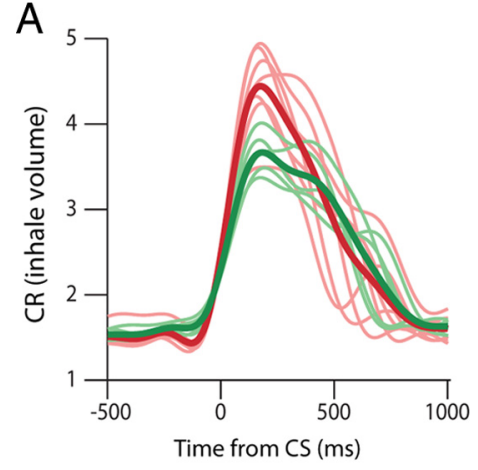

C

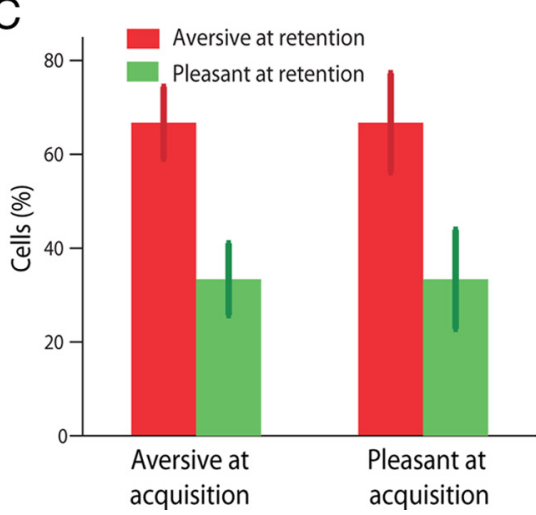

B
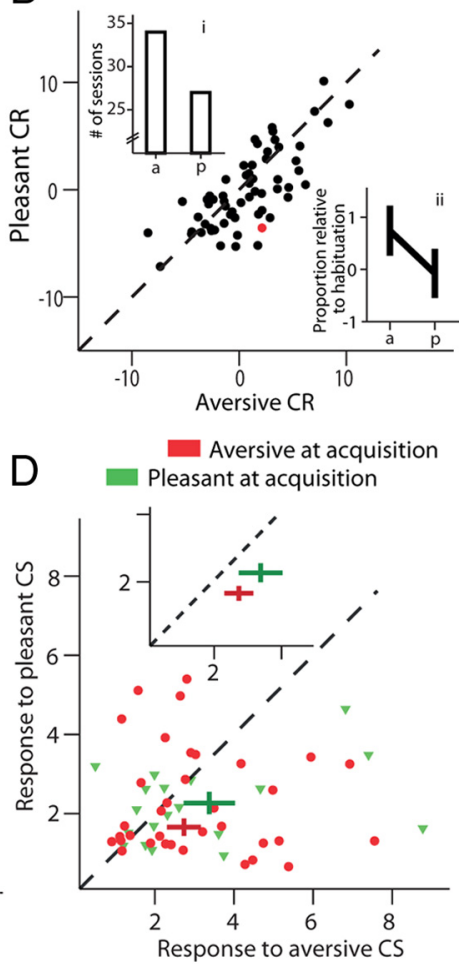

Figure 9. $24 \mathrm{~h}$ (overnight) retention reveals an aversive-bias in neural responses. $\boldsymbol{A}$, Mean CR overlaid by individual traces (thin lines) in the retention test of one session, i.e., the CS are the tones from the previous day acquisition. $B$, Mean (R from all sessions for ACS versus PCS indicates memory for valence discrimination i.e., higher CR for ACS (red point corresponds to the session shown in $\boldsymbol{A}$. Insets show the difference between sessions above the identity line versus below the identity line in two ways: the number of sessions (inset $i ; p<0.05, \chi^{2}$ ), and the mean inhale-volume relative to habituation (inset $i i ; p<0.05, t$ tests). C, Proportion of cells with aversive-selectivity (red) or pleasant-selectivity (green) during (S-evoked responses in retention, of cells that had aversive-selectivity (left) or pleasant-selectivity (right) during (S-evoked responses in the acquisition stage. This reveals a clear aversive bias in cells' selectivity ( $p<0.05$, binomial test; error bars represent $\pm 95 \%$ binomial confidence interval). $D, M o r e o v e r$, both pleasant-selective and aversive-selective cells during acquisition had a significantly stronger average response for aversive CS during retention (inset shows populations mean $\pm \mathrm{SEM}, p<0.05$ for both, $t$ tests).

session (for 2 new tones). We found that $22 \%$ of responsive cells (44/199, $p<0.01$, binomial test) discriminated between aversive and pleasant tones during retention, but there was a higher ratio for aversive-selectivity among cells that later had either aversive- or pleasant-selectivity during acquisition (Fig. 9C, 66\% in both, $p<0.05$ for both, binomial test). In addition, the peak responses for aversive CS during retention were significantly higher than for pleasant CS, and this was so independent of the valence-selectivity that the cell had during the following acquisition session (Fig. 9D; $p<0.05, t$ tests). To further confirm that this result is due to valence rather than intensity (odors were matched for intensity in preliminary experiments with human subjects, $p>0.1, t$ test), we repeated all analyses on a subset of days (35/76, 125 neurons recorded in these sessions) in which the absolute magnitude of breath modulation was similar for aversive and pleasant odors ( $t$ test, $p>0.05$ ). The aversive-bias effect was replicated $(p<0.05$ for all tests as in Fig. 9C,D), suggesting that the main contributor is valence.

The overall results suggest a mild, yet significant, tendency for aversive representation for long-term (overnight) association memories.

\section{Discussion}

Our results supply the first electrophysiological description of how neurons in the primate amygdala are involved in acquisition and extinction learning, and in overnight retention of pleasant and aversive memories. We developed a paradigm using tone-odor associations and identified distinct neural populations that specifically code for acquisition or extinction and that are valenceselective. We found that neurons fire at synchrony with respiratory cycles in a stage-dependent manner that is enhanced during acquisition. We find that different neurons signal for 
positive valence, negative valence, or both (generalizing), and we identify two types of aversive bias. One such bias occurs during acquisition and formation of associations when neurons that originally respond to pleasant US become responsive to the aversive CS (but much less so for the opposite direction). Another type of aversive bias occurs after $24 \mathrm{~h}$ (overnight), when neurons tend to respond more to the aversive CS of the previous day, although they might later acquire positive-selective responses (and again, much less so for the opposite direction). Below, we discuss our findings in relation to other rodent and primate studies.

We found that spontaneous firing in the BLA is synchronized with respiratory cycles, and that there is increased temporal locking and response magnitude during the acquisition stage. Breath-locked cells were homogenously distributed across the BLA ( $p>0.1, \chi^{2}$, data not shown), suggesting that it is not due to possible few recordings from the CeA or other structures. Moreover, we demonstrate that stimuli-evoked responses can be observed only when contrasted against spontaneous respiratory-related activity. This implies that decoding occurs in downstream neurons that receive autonomic information, as in the CeA that has reciprocal connection with the brainstem and hypothalamus. A previous study reported that neurons in the human BLA are locked to respiration, but in much smaller numbers (Frysinger and Harper, 1989), and this might indicate that the higher numbers we observed are related to the actual paradigm we used — one that is dependent on breathing and its cycle.

This, and our finding that locking is modulated by stage, implies that the BLA is dynamically modulated by emotional state. An alternative interpretation, which is not mutually exclusive, is that it reflects increased attention during acquisition (Gallagher and Holland, 1994; Davis and Whalen, 2001; Roesch et al., 2010). Nevertheless, our results show that this modulation is not exhibited by a general tonic increase, but is specific to the respiratory nature of the task, which might suggest involvement of inputs from regions that participate in active sniffing (Sobel et al., 1998; Shusterman et al., 2011), or by noradrenergic innervations from the nucleus of the solitary tract (NTS; Clayton and Williams, 2000), either directly or indirectly via the thalamus or the insula. A recent study in monkeys demonstrated locking to skin conductance responses (SCR) - another autonomic response-in BLA neurons, even in the absence of external stimuli (Laine et al., 2009). The combined findings suggest that neurons in the BLA are engaged in coding the current emotional or attentional state of the animal as it changes along the different learning stages (Murray, 2007; Morrison and Salzman, 2010).

These stage-dependent changes in spontaneous firing rates can serve a double purpose. They can regulate signal-to-noise ratio and dynamic sensitivity by providing different offsets for CS-evoked phasic responses, either in BLA neurons themselves or in their targets CeA neurons, as was recently found in the rodent amygdala (Ciocchi et al., 2010). This was specifically demonstrated here by neurons that reduced their activity when compared with their elevated baseline breathing response. In parallel, they can facilitate learning-dependent plasticity (e.g., LTP) and population synchrony by providing focused elevated activity in crucial times of behavior (Shusterman et al., 2011), i.e., when expecting an event with emotional significance. In our paradigm, odor-release was always synchronized with inhale onset, and we indeed found higher activations and more neurons with peak firing rates at the first half of the respiratory cycle-the inhale stage.

We found that different subgroups of neurons code for acquisition and for extinction, and reconstruction of electrode locations suggests that these two groups are homogenously intermingled within the BLA $\left(p>0.1, \chi^{2}\right)$. This is similar to recent findings in the rodent amygdala (Herry et al., 2008), and we extend this here to show that this is the case also for acquisition and extinction of positive stimuli (Tye et al., 2010). We also found that neurons that selectively respond to either valence of the reinforcers (odors) and to acquired valence (i.e., the CS), are also homogenously intermingled in the BLA (data not shown). All in all, it seems the primate amygdala is equally important for coding of both positive and negative associated cues (Paton et al., 2006), and this view receives further support from studies that show coding for relative complex facets of positive stimuli as its magnitude (Bermudez and Schultz, 2010), and expectancy (Fuster and Uyeda, 1971; Schoenbaum et al., 1998; Sugase-Miyamoto and Richmond, 2005; Belova et al., 2007). Our observation of significant number of generalizing neurons that code for both types of valence is consistent with literature linking the amygdala with evaluation of intensity (Anderson et al., 2003; Winston et al., 2005) or absolute value (Morrison and Salzman, 2010; Roesch et al., 2010).

In addition to homogenous coding of value, we show that there are two types of aversive-bias. The first, learning-related bias was observed when comparing unconditioned responses for the US and acquired responses for the CS. This revealed that there is a higher tendency for neurons that code for positive reinforcer to acquire responses for the aversive CS than vice versa. The second bias was revealed when comparing responses for the previous day associations to responses for the new associations formed in the session. These two types of bias suggest that although the amygdala might equally evaluate aversive and pleasant cues, it selectively potentiates aversive-related information for the longer-term. Such overnight plasticity can induce downstream changes, as was recently observed in Ce neurons (Duvarci et al., 2011). Our results can therefore reconcile previous notions that regarded the amygdala as the "fear center" of the brain, mainly due to the influential work on aversive classical conditioning (LeDoux, 2000), with the wealth of aforementioned findings that associated the amygdala with processing of positive stimuli, and the recently emerging view that the amygdala is equally important in evaluating both types of valence (Baxter and Murray, 2002; Murray, 2007; Tye et al., 2008; Morrison and Salzman, 2010). Our study uses the same modality to confirm that the amygdala is important for the processing of negative and positive contingencies, yet to show that negative valence can induce further plasticity for longer-term memory. This provides further insight to why the amygdala is implicated in traumatic memories (Milad et al., 2006; Shin et al., 2006).

Several studies have recently shown that immediate extinction-training results in less extinction consolidation and higher levels of the original memory in the following day (Maren and Chang, 2006; Schiller et al., 2008; Woods and Bouton, 2008). We replicated and used this here. Because we did not introduce sessions without extinction and because comparing breathing across days is variable and very contextdependent, it is hard to conclude whether the memory has recovered completely or whether it is still somewhat diminished. However, our behavior clearly shows that extinction was complete in the same day and that there was clear memory 
(spontaneous recovery) of the associations in the following day. This allowed us to investigate the activity of single neurons in the primate amygdala in relation to both effects, and provide first evidence to the dynamics of coding during extinction and long-term (overnight) retention of pleasant and aversive memories.

\section{References}

Amano T, Unal CT, Paré D (2010) Synaptic correlates of fear extinction in the amygdala. Nat Neurosci 13:489-494.

Amaral DG, Price JL (1984) Amygdalo-cortical projections in the monkey (Macaca fascicularis). J Comp Neurol 230:465-496.

Anderson AK, Christoff K, Stappen I, Panitz D, Ghahremani DG, Glover G, Gabrieli JD, Sobel N (2003) Dissociated neural representations of intensity and valence in human olfaction. Nat Neurosci 6:196-202.

Antoniadis EA, Winslow JT, Davis M, Amaral DG (2009) The nonhuman primate amygdala is necessary for the acquisition but not the retention of fear-potentiated startle. Biol Psychiatry 65:241-248.

Baxter MG, Murray EA (2002) The amygdala and reward. Nat Rev Neurosci 3:563-573.

Belova MA, Paton JJ, Morrison SE, Salzman CD (2007) Expectation modulates neural responses to pleasant and aversive stimuli in primate amygdala. Neuron 55:970-984.

Bermudez MA, Schultz W (2010) Reward magnitude coding in primate amygdala neurons. J Neurophysiol 104:3424-3432.

Carmichael ST, Clugnet MC, Price JL (1994) Central olfactory connections in the macaque monkey. J Comp Neurol 346:403-434.

Ciocchi S, Herry C, Grenier F, Wolff SB, Letzkus JJ, Vlachos I, Ehrlich I, Sprengel R, Deisseroth K, Stadler MB, Müller C, Lüthi A (2010) Encoding of conditioned fear in central amygdala inhibitory circuits. Nature 468:277-282.

Clayton EC, Williams CL (2000) Adrenergic activation of the nucleus tractus solitarius potentiates amygdala norepinephrine release and enhances retention performance in emotionally arousing and spatial memory tasks. Behav Brain Res 112:151-158.

Davis M, Whalen PJ (2001) The amygdala: vigilance and emotion. Mol Psychiatry 6:13-34.

Delgado MR, Olsson A, Phelps EA (2006) Extending animal models of fear conditioning to humans. Biol Psychol 73:39-48.

Duvarci S, Popa D, Paré D (2011) Central amygdala activity during fear conditioning. J Neurosci 31:289-294.

Fanselow MS, Poulos AM (2005) The neuroscience of mammalian associative learning. Annu Rev Psychol 56:207-234.

Frysinger RC, Harper RM (1989) Cardiac and respiratory correlations with unit discharge in human amygdala and hippocampus. Electroencephalogr Clin Neurophysiol 72:463-470.

Fuster JM, Uyeda AA (1971) Reactivity of limbic neurons of the monkey to appetitive and aversive signals. Electroencephalogr Clin Neurophysiol 30:281-293.

Gallagher M, Holland PC (1994) The amygdala complex: multiple roles in associative learning and attention. Proc Natl Acad Sci USA 91:11771-11776.

Ghashghaei HT, Hilgetag CC, Barbas H (2007) Sequence of information processing for emotions based on the anatomic dialogue between prefrontal cortex and amygdala. Neuroimage 34:905-923.

Gothard KM, Battaglia FP, Erickson CA, Spitler KM, Amaral DG (2007) Neural responses to facial expression and face identity in the monkey amygdala. J Neurophysiol 97:1671-1683.

Gottfried JA, O'Doherty J, Dolan RJ (2002) Appetitive and aversive olfactory learning in humans studied using event-related functional magnetic resonance imaging. J Neurosci 22:10829-10837.

Haubensak W, Kunwar PS, Cai H, Ciocchi S, Wall NR, Ponnusamy R, Biag J, Dong HW, Deisseroth K, Callaway EM, Fanselow MS, Lüthi A, Anderson DJ (2010) Genetic dissection of an amygdala microcircuit that gates conditioned fear. Nature 468:270-276.

Herry C, Ciocchi S, Senn V, Demmou L, Müller C, Lüthi A (2008) Switching on and off fear by distinct neuronal circuits. Nature 454:600-606.

Izquierdo A, Murray EA (2005) Opposing effects of amygdala and orbital prefrontal cortex lesions on the extinction of instrumental responding in macaque monkeys. Eur J Neurosci 22:2341-2346.

Kuraoka K, Nakamura K (2007) Responses of single neurons in monkey amygdala to facial and vocal emotions. J Neurophysiol 97:1379-1387.
Laine CM, Spitler KM, Mosher CP, Gothard KM (2009) Behavioral triggers of skin conductance responses and their neural correlates in the primate amygdala. J Neurophysiol 101:1749-1754.

LeDoux JE (2000) Emotion circuits in the brain. Annu Rev Neurosci 23:155-184.

Likhtik E, Popa D, Apergis-Schoute J, Fidacaro GA, Paré D (2008) Amygdala intercalated neurons are required for expression of fear extinction. Nature 454:642-645.

Livneh U, Paz R (2010) An implicit measure of olfactory performance for non-human primates reveals aversive and pleasant odor conditioning. J Neurosci Methods 192:90-95.

Maren S, Chang CH (2006) Recent fear is resistant to extinction. Proc Natl Acad Sci U S A 103:18020-18025.

Maren S, Quirk GJ (2004) Neuronal signalling of fear memory. Nat Rev Neurosci 5:844-852.

Martin RF, Bowden DM (2000) Primate brain maps: structure of the macaque brain. Amsterdam, The Netherlands: Elsevier Science.

McDonald AJ (1998) Cortical pathways to the mammalian amygdala. Prog Neurobiol 55:257-332.

McGaugh JL (2004) The amygdala modulates the consolidation of memories of emotionally arousing experiences. Annu Rev Neurosci 27:1-28.

Milad MR, Quirk GJ (2002) Neurons in medial prefrontal cortex signal memory for fear extinction. Nature 420:70-74.

Milad MR, Rauch SL, Pitman RK, Quirk GJ (2006) Fear extinction in rats: implications for human brain imaging and anxiety disorders. Biol Psychol 73:61-71.

Morrison SE, Salzman CD (2010) Re-valuing the amygdala. Curr Opin Neurobiol 20:221-230.

Mosher CP, Zimmerman PE, Gothard KM (2010) Response characteristics of basolateral and centromedial neurons in the primate amygdala. J Neurosci 30:16197-16207.

Murray EA (2007) The amygdala, reward and emotion. Trends Cogn Sci 11:489-497.

Myers KM, Davis M (2007) Mechanisms of fear extinction. Mol Psychiatry 12:120-150.

Nishijo H, Ono T, Nishino H (1988) Single neuron responses in amygdala of alert monkey during complex sensory stimulation with affective significance. J Neurosci 8:3570-3583.

Ochsner KN, Gross JJ (2005) The cognitive control of emotion. Trends Cogn Sci 9:242-249.

Pape HC, Paré D (2010) Plastic synaptic networks of the amygdala for the acquisition, expression, and extinction of conditioned fear. Physiol Rev 90:419-463.

Paré D, Quirk GJ, Ledoux JE (2004) New vistas on amygdala networks in conditioned fear. J Neurophysiol 92:1-9.

Paton JJ, Belova MA, Morrison SE, Salzman CD (2006) The primate amygdala represents the positive and negative value of visual stimuli during learning. Nature 439:865-870.

Paz R, Pelletier JG, Bauer EP, Paré D (2006) Emotional enhancement of memory via amygdala-driven facilitation of rhinal interactions. Nat Neurosci 9:1321-1329.

Phelps EA, Delgado MR, Nearing KI, LeDoux JE (2004) Extinction learning in humans: role of the amygdala and vmPFC. Neuron 43:897-905.

Quirk GJ, Beer JS (2006) Prefrontal involvement in the regulation of emotion: convergence of rat and human studies. Curr Opin Neurobiol $16: 723-727$.

Roesch MR, Calu DJ, Esber GR, Schoenbaum G (2010) Neural correlates of variations in event processing during learning in basolateral amygdala. J Neurosci 30:2464-2471.

Sanghera MK, Rolls ET, Roper-Hall A (1979) Visual responses of neurons in the dorsolateral amygdala of the alert monkey. Exp Neurol 63:610-626.

Schiller D, Cain CK, Curley NG, Schwartz JS, Stern SA, Ledoux JE, Phelps EA (2008) Evidence for recovery of fear following immediate extinction in rats and humans. Learn Mem 15:394-402.

Schoenbaum G, Chiba AA, Gallagher M (1998) Orbitofrontal cortex and basolateral amygdala encode expected outcomes during learning. Nat Neurosci 1:155-159.

Shin LM, Rauch SL, Pitman RK (2006) Amygdala, medial prefrontal cortex, and hippocampal function in PTSD. Ann N Y Acad Sci 1071:67-79.

Shusterman R, Smear MC, Koulakov AA, Rinberg D (2011) Precise olfactory responses tile the sniff cycle. Nat Neurosci 14:1039-1044. 
Sobel N, Prabhakaran V, Desmond JE, Glover GH, Goode RL, Sullivan EV, Gabrieli JD (1998) Sniffing and smelling: separate subsystems in the human olfactory cortex. Nature 392:282-286.

Sotres-Bayon F, Bush DE, LeDoux JE (2007) Acquisition of fear extinction requires activation of NR2B-containing NMDA receptors in the lateral amygdala. Neuropsychopharmacology 32:1929-1940.

Sugase-Miyamoto Y, Richmond BJ (2005) Neuronal signals in the monkey basolateral amygdala during reward schedules. J Neurosci 25:1107111083.

Tsoory MM, Vouimba RM, Akirav I, Kavushansky A, Avital A, Richter-Levin G (2008) Amygdala modulation of memory-related processes in the hippocampus: potential relevance to PTSD. Prog Brain Res 167:35-51.
Tye KM, Stuber GD, de Ridder B, Bonci A, Janak PH (2008) Rapid strengthening of thalamo-amygdala synapses mediates cue-reward learning. Nature 453:1253-1257.

Tye KM, Cone JJ, Schairer WW, Janak PH (2010) Amygdala neural encoding of the absence of reward during extinction. J Neurosci 30:116-125.

Winston JS, Gottfried JA, Kilner JM, Dolan RJ (2005) Integrated neural representations of odor intensity and affective valence in human amygdala. J Neurosci 25:8903-8907.

Woods AM, Bouton ME (2008) Immediate extinction causes a less durable loss of performance than delayed extinction following either fear or appetitive conditioning. Learn Mem 15:909-920. 